\title{
Prevention of MSD within OHSMS/IMS: a systematic review of risk assessment strategies
}

\author{
Amin Yazdani ${ }^{\mathrm{a},{ }^{*}}$ and Richard Wells ${ }^{\mathrm{a}, \mathrm{b}}$ \\ ${ }^{a}$ Department of Kinesiology, University of Waterloo, Waterloo, Ontario, Canada \\ ${ }^{\mathrm{b}}$ Centre of Research Expertise for the Prevention of Musculoskeletal Disorders (CRE-MSD), Ontario, Canada
}

\begin{abstract}
The purpose of this systematic review was to identify and summarize the research evidence on prevention of Musculoskeletal Disorders (MSD) within Occupational Health and Safety Management Systems (OHSMS) and Integrated Management Systems (IMS). Databases in business, management, engineering and health and safety were systematically searched and relevant publications were synthesized. The number of papers that could address the research questions was small. However, the review revealed that many of the techniques to address MSD hazards require substantial background knowledge and training. This may limit employees' involvement in the technical aspects of the risk assessment process. Also these techniques did not usually fit into techniques used by companies to address other risk factors within their management systems. This could result in MSD prevention becoming a separate issue that cannot be managed with company-wide tools. In addition, this review also suggested that there is a research gap concerning the MSD prevention within companies' management systems.
\end{abstract}

Keywords: Occupational health and safety management systems, integrated management systems, musculoskeletal disorders, risk assessment, ergonomics

\section{Introduction}

A "management system" is the framework of individual processes, procedures and resources joined to ensure achievement of certain objectives effectively and efficiently [5]. To do this, several management system standards and guidelines have been developed. The three main internal management systems including "environmental management system" (EMS), "quality management system" (QMS) and "occupational management system" (OHSMS) have common structure and requirements. These management systems can be integrated so that both the costs of implementation and the running costs can be reduced. This is an "Integrated Management System" (IMS)[1]. Risk Analysis is the main common requirement for these management systems [6]. In order to comply with standard requirements, companies should systematically manage different type of risk factors within their workplace including chemical, physical, mechanical, biological, environmental, ergonomic and quality risk factors. On the other hand, the prevalence of musculoskeletal disorders (MSD) is still high within workplaces. This may be due to the fact that MSD hazards are not being addressed as effectively as possible, because (ergonomic) MSD hazard assessment, and by extension prevention, is partially outside the main management processes. Information concerning MSD hazards may therefore not be "onthe-table" and so may not receive adequate attention. An approach which could facilitate bringing ergonomics into companies' management systems could be very valuable. This paper will systematically review literature on risk assessment methods used within company's management systems. This review paper aims to document how the literature addressed MSD risk assessment tools and strategies used to incorporate them into management systems.

\footnotetext{
* Corresponding author: Amin Yazdani, Department of Kinesiology, University of Waterloo, 200 University Avenue, W., Waterloo, Ontario, Canada, N2L 3G1. Tel. No.: 519-888-4567 ext. 31396. E-mail: ayazdani@uwaterloo.ca
} 


\section{Methods}

\subsection{Systematic review process}

The process included following sequential steps: 1) the research questions were clearly identified; 2) the inclusion and exclusion criteria were described; 3) search schemes were defined; 4) the extensive literature search were conducted; 5) relevant studies were selected; 6) the evidence was extracted and 7) the final result of systematic review were summarized.

\subsection{Literature search for identification of studies}

Electronic data bases that were systematically searched for relevant documents included MEDLINE, EMBASE, Compendex, Web of Science, PsycINFO, Ergonomic Abstracts, and other 44 data bases using ProQuest search platform. The search strategy combined two sets of keywords using the Boolean operator "AND" while "OR" strategy was used to combine the keywords within each group. In addition, the reference list of documents were manually searched and included, if met the selection criteria. The first set of keywords were focused on following terms: musculoskeletal disorders (MSD), ergonomics, low back pain, cumulative trauma disorders (CTD), upper extremities, repetitive strain injuries (RSI), musculoskeletal injuries (MSI), injury prevention. The following keywords were used as the second set for management systems: occupational health and safety management system, health and safety management system, integrated management system, quality management system, total quality management system, risk assessment, and risk management. The keywords were searched in title, abstracts, and topics.

\subsection{Document relevance review}

The title, keywords and abstract of each abstract were reviewed by the researchers. Relevant articles with respect to following criteria were retrieved. Peer reviewed journal articles, conference papers, and related articles about practices in any management systems were included. Both qualitative and quantitative studies that had addressed the research question were included. However, studies that didn't provide information about risk assessment techniques and strategies and also those documents not in English were excluded.

\subsection{Data extraction and synthesis of information}

The reviewers extracted data from documents about context, type of risk assessment techniques, strategies used, techniques within company's management system and any authors' comments and recommendations about MSD prevention within a company's management system. The thematic synthesis technique was used to combine the findings. This technique, which is being used in the analysis of qualitative data, systematically identifies the main and the most important themes based on the research question [8].

\section{Results and discussion}

\subsection{Risk assessment techniques within company's management systems}

It has been stated that an appropriate risk assessment process is needed to guarantee an effective approach to health and safety at work [3]. Case studies in implementing IMS using the risk analysis based approach were described by Labodova [6]. In companies with and without systematic management system suggested this approach can be used in any kind of company including SME's with proper training. The risk assessment methodology introduced by that author had both qualitative and quantitative aspects of risk assessment. The risk matrix developed was based on a common scale of financial acceptability to compare levels in different areas (quality, environmental, health and safety) in IMS and was the result of a top management decision.

A comprehensive study by Tixier et al [13] reported on 62 qualitative and quantitative risk analysis methodologies that have been used widely in industries for different type of risks. The International Standard Organization (ISO) introduces general guidance across many industries and type of systems. ISO 31000 was published in 2009 to provide information on the implementation of risk management [4]. Techniques such as HAZard and OPerability (HAZOP), Layer Of Protection Analysis (LOPA) and Failure Mode and Effects Analysis (FMEA) are examples of widely used techniques described by ISO 31000. In terms of integrating MSD prevention into techniques described by ISO, very little work has been reported. Shephard et al [11] described the development of an Ergonomic FMEA for use in design and Munck-Ulfsfält [9] described a similar approach. 
These are the only attempts reported in literature that aim to harmonize ergonomic assessments with common risk assessment methods used in design, but not however in OHSMS/IMS.

\subsection{MSD hazard assessment techniques}

Numerous methods have been developed to assess biomechanical exposure for musculoskeletal disorders [2, 12]. Takala et al [12] systematically evaluated 29 observational methods assessing biomechanical exposure at work including the Washington State Ergonomic checklist, Ovako Working Posture Assessment (OWAS), Quick Exposure Check (QEC), Posture Activity Tools and Handling (PATH), NIOSH Lifting Equation, ACGIH lifting TLV (USA), ManTRA (Australia), NZ Code for Material Handling (New Zealand), and etc. Many of these techniques require substantial background knowledge and training to accurately assess MSD hazards. This limits workers' involvement in the technical aspects of the risk assessment process. Interestingly, despite the ISO 31000 Standard being introduced in 2009, there is no mention of (ergonomic) risk assessment techniques relevant to MSD in this standard.

\subsection{Company's specific tools and strategies}

There are many types of risk assessment techniques and strategies developed by individual companies to address MSD hazards. As an example, Cohen [1] described a program developed by an electronic manufacturer in California, US to prevent Repetitive Strain Injuries (RSI). It was implemented by 4 different sub-committees (teams) working under a larger committee. In this program, the supervisor and manager are ultimately accountable to implement and follow-up on corrective actions. Cohen concludes by stating that this program, particularly with worker involvement, significantly reduced RSI severity. The program was noted to be a part of the company's management system but there was no mention of a systematic risk management strategy with a continual improvement and management review commitment.

\section{Conclusion}

Currently there are many published methods and company specific MSD hazard identification assessment methods available. However the literature is silent on how to use these effectively with manage- ment systems. These systems require that data be collected, analyzed and displayed in a consistent manner. Hence, it is important to link methods used in MSD prevention activities to management systems and make ergonomics an "everyday tool" in workplaces and in design departments [7]. To help this happen, MSD assessment terminology, data and reporting should be translated into the common language used in company-wide management systems. The number of published works in this area is small but it clearly showed a research gap and the need for developing an approach to integrate MSD prevention into management systems. This would help avoid health and safety, and especially MSD prevention, becoming a "sidecar" function [10] and reducing the effectiveness of MSD prevention activities.

\section{References}

[1] R. Cohen, Ergonomics program development: Prevention in the workplace. AIHA J, 58(2) (1997), 145-149.

[2] P.G. Dempsey, R.W. McGorry, and W.S. Maynard, A survey of tools and methods used by certified professional ergonomists. Applied Ergonomics. 36(4) (2005), 489-503.

[3] M. Fera, and R. Macchiaroli, Appraisal of a new risk assessment model for SME, Safety Sci, 48(10) (2010), 1361-1368.

[4] International Standard Organization, ISO 31000:2009. Retrieved March 21, 2011, from: http://www.iso.org/iso/catalogue_detail.htm?csnumber $=43170$

[5] S. Karapetrovic, W. Willborn, Connecting internal management systems in service organizations, Managing Service Quality, 8(4) (1998), pp.256 - 271.

[6] A. Labodová, Implementing integrated management systems using a risk analysis based approach, Journal of Cleaner Production, 12(6) (2004), 571-580.

[7] K.S. Lee, Ergonomics in total quality management: How can we sell ergonomics to management?, Ergonomics, 48(5) (2005), 547-558.

[8] N. Mays, C. Pope, J. Popay, Systematically reviewing qualitative and quantitative evidence to inform management and policy-making in the health field, $J$ Health Serv Res Policy, 10 (suppl 1) (2005), 6-20.

[9] U. Munck-Ulfsfalt, A. Falck, A. Forsberg, C. Dahlin, A. Eriksson, Corporate ergonomics programme at Volvo Car Corporation, Applied Ergonomics, 34 (2003), 17-22.

[10] W.P. Neumann, and J. Dul, Workshop Report: Ergonomics' contributions to company strategies. Paper presented at the Nordic Ergonomics Society (NES) - 37th Annual Conference, Oslo, Norway (2005)

[11]D.J. Shephard, D. Villalta, J.R. Potvin, A system to incorporate ergonomics into product design and processes for manufacturing assembly, in: Proceedings of the Association of the Canadian Ergonomist Conference, London, Ontario (2003).

[12]E.P. Takala, I. Pehkonen, M. Forsman, G.A. Hansson, S.E. Mathiassen, W.P. Neumann, et al, Systematic evaluation of observational methods assessing biomechanical exposures at work, Scand J Work Environ Health, 36(1) (2010), 3-24.

[13]J. Tixier, J, G. Dusserre, O. Salvi, and D. Gaston, Review of 62 risk analysis methodologies of industrial plants, $J$ of Loss Prevention in the Process Industries, 15(4) (2002), 291-303. 